OPEN ACCESS

Edited by:

Christian Michael Hedrich University of Liverpool,

United Kingdom

Reviewed by:

Jillian M. Richmond,

University of Massachusetts Medical

School, United States

Andras Perl,

Upstate Medical University,

United States

*Correspondence:

Juan Shan

shanjuan858@foxmail.com

tThese authors have contributed equally to this work

Specialty section:

This article was submitted to Autoimmune and Autoinflammatory

Disorders,

a section of the journal

Frontiers in Immunology

Received: 11 March 2020 Accepted: 29 April 2020

Published: 22 May 2020

Citation:

Shan J, Jin H and Xu Y (2020) T Cell Metabolism: A New Perspective on Th17/Treg Cell Imbalance in Systemic Lupus Erythematosus.

Front. Immunol. 11:1027. doi: 10.3389/fimmu.2020.01027

\section{T Cell Metabolism: A New Perspective on Th17/Treg Cell Imbalance in Systemic Lupus Erythematosus}

\author{
Juan Shan ${ }^{\star t}$, Hong Jin ${ }^{\dagger}$ and Yan $\mathrm{Xu}^{+}$ \\ Department of Immunology, School of Basic Medical Sciences, Chengdu Medical College, Chengdu, China
}

The Th17/T-regulatory (Treg) cell imbalance is involved in the occurrence and development of organ inflammation in systemic lupus erythematosus (SLE). Metabolic pathways can regulate $T$ cell differentiation and function, thus contributing to SLE inflammation. Increasingly, data have shown metabolism influences and reprograms the Th17/Treg cell balance, and the metabolic pattern of T cells is different in SLE. Notably, metabolic characteristics of SLE T cells, such as enhanced glycolysis, lipid synthesis, glutaminolysis, and highly activated mTOR, all favored Th17 differentiation and function, which underlie the Th17/Treg cell imbalance in SLE patients. Targeting metabolic pathways to reverse Th17/Treg imbalance offer a promising method for SLE therapy.

Keywords: cell metabolism, T helper 17 cells, regulatory T cells, systemic lupus erythematosus, mTOR signaling

\section{INTRODUCTION}

Systemic lupus erythematosus (SLE) is an autoimmune disease characterized by tissue inflammation and profound damage to multiple organs. The pathological mechanisms of SLE remain unclear; however, it has been reported that the imbalance between T helper 17 (Th17) and regulatory T cells (Tregs) underlies the pathogenesis of SLE (1-4). Th17 cells have proinflammatory effects, and the proportion of Th17 cells is higher in SLE patients, and the content is positively correlated with the activity of SLE disease (5). Tregs, however, have immunosuppressive function and play an important role in the induction and maintenance of self-tolerance. The reduced content and dysfunction of Tregs are closely related to the occurrence and development of SLE (6-8). Tregs injected into lupus mice can control the inflammatory response and alleviate pathological damage (9). Thus, improving the Th17/Treg cells imbalance shows some promise for the treatment of SLE.

Th17 and Treg cells are mutually antagonistic in function and differentiation. Many mechanisms regulating Th17/Treg cells balance have been reported. Besides the molecular signaling network, accumulating evidence has shown that cellular metabolism is also critically involved in Th17 and Treg differentiation (10). These two cell subsets are dictated by distinct metabolic pathways, and manipulating metabolic pathways can regulate Th17/Treg cells balance. In Tcells from SLE patients and lupus-prone mice, metabolic abnormalities linked to Th17/Treg cell imbalances have been reported. Here, we reviewed how cellular metabolism influences Th17 and Treg cell differentiation, summarized metabolic abnormalities of SLE T cells, 
and we here propose that metabolic abnormalities of SLE T cells is the mechanism by which the Th17/Treg imbalance emerges in SLE patients. Manipulating cellular metabolism to correct aberrant immune responses may be a suitable means of treating SLE.

\section{METABOLIC CONTROL OF Th17/Treg BALANCE}

T-cell metabolism is highly dynamic. The metabolic pattern changes during the process of activation, proliferation, and differentiation. Naive $\mathrm{T}$ cells have low energy requirements. They import a small amount of glucose and generate ATP mainly through the TCA cycle and OXPHOS. Upon activation, $\mathrm{T}$ cells start to proliferate and differentiate. They need to reprogram their metabolic pattern to meet their bioenergetic and biosynthetic requirements. A variety of metabolic substrates (glucose, amino acids, and fatty acids) and metabolic pathways (glycolysis, oxidative phosphorylation, the pentose phosphate pathway, fatty acid synthesis and oxidation, and glutamine metabolism) are mobilized to adapt to their biological functions. Glycolysis can rapidly produce ATP to provide the energy needed to rapidly proliferate, and a large number of intermediate products of various metabolic pathways allow biosynthesis (11-14).

Studies have shown that $\mathrm{T}$ helper cell subsets have different metabolic patterns, of which those of Th17 cells and Tregs are the most distinct. One study evaluated 400 energy metabolites, metabolism-related genes, and proteins in Th17 and Tregs and results indicated that Th17 cells contain high levels of pyruvate, lactic acid, early glycolysis, and pentose phosphate pathway intermediates, and they express key proteins in glycolysis pathway, like Glut-1 and HK-2, at high levels. Tregs had higher $\mathrm{C} 2$ and $\mathrm{C} 4-\mathrm{OH}$ carnitine levels and more expression of fatty acid transporter CPT1A and electron transport chain component cytochrome c, which suggested that Th17 cells were mainly powered by glycolysis, and the pentose phosphate pathway was also active; Tregs, on the other hand, were found to rely more on fatty acid oxidation and oxidative phosphorylation to supply energy (15). Glycolysis deprivation was found to impair Th17 differentiation dramatically, while defective glycolysis supported the development of Treg cells. Replacement of glucose with galactose, treatment with 2-DG (an inhibitor of hexokinase, the first rate-limiting enzyme of glycolysis), and lack of HIF1 $\alpha$, Cdc42, ICER, and mTOR (crucial regulators of $\mathrm{T}$ cell glycolytic metabolism) all resulted in diminished Th17 development but enhanced Treg cell differentiation (16-20). Conversely, inhibition of fatty acid oxidation results in diminished differentiation to Th17 cells, but increased development of Tregs (21).

Glutaminolysis is also preferentially increased in Th17 cells. TCA-cycle intermediates produced after $\alpha$-ketoglutarate $(\alpha-K G)$ in Th17 cells were more plentiful than in Tregs. $\alpha-k G$ is also a metabolite of glutamine (15), suggesting increased glutaminolysis in Th17 cells. ICER, a transcriptional factor that enhances glutaminase 1 and promotes glutaminolysis, is also expressed in large quantities in Th17 cells (22). In addition, glutamine metabolite 2-hydroxyglutarate could hypermethylate Foxp3 gene locus and inhibit Foxp3 transcription, thus promoting the differentiation of Th17 cells, which regulates Th17/Treg balance by an epigenetic mechanism (23).

Th17 cells have considerable fatty acid synthesis activity. The expression of ACC1, a key enzyme in fatty acid synthesis, was found to be significantly higher in Th17 cells than in Tregs. Drug inhibition or T-cell specific knockout of ACC1 could inhibit Th17 differentiation and promote the induction of Tregs both in vivo and in vitro $(24,25)$. Cholesterol intake and synthesis were also significantly higher in Th17 cells (26), leading to the accumulation of the cholesterol precursor, desmosterol, which acts as a potent endogenous $\operatorname{ROR} \gamma$ agonist and dictates Th17 differentiation (27). Statins, a class of drugs that inhibit cholesterol biosynthesis, are reported to target Th17/Treg imbalance and alleviate Th17-mediated inflammatory response (28). The distinct metabolic patterns of Th17 and Treg cells provide a basis for intervention of Th17/Treg imbalance.

\section{METABOLIC ABNORMALITIES IN SLE T CELLS}

Cell metabolism regulates the differentiation and function of $\mathrm{T}$ cells, thereby participating in the occurrence and development of SLE inflammation. Metabolic abnormalities in T cells from SLE patients and lupus-prone mice were reported $(29,30)$. These are characterized by the following:

(1) Mitochondrial dysfunction: T cells from SLE patients showed elevated mitochondrial transmembrane potential, increased ROS production and reduced ATP synthesis (31).

(2) Hyperactivated glucose metabolism: $\mathrm{CD} 4^{+} \mathrm{T}$ cells from SLE patients and lupus-prone mice have higher OCR and ECAR levels $(32,33)$, suggesting they have elevated levels of both glycolysis and oxidative phosphorylation. The metabolites of pentose phosphate pathway, such as R5P and F6P, were also higher in peripheral blood lymphocytes of SLE patients (34). These results suggested that three main pathways of glucose metabolism - aerobic glycolysis, pentose phosphate pathway, and oxidative phosphorylation-are involved in $\mathrm{T}$ cell activation in SLE patients.

(3) Lipid synthesis enhancement: there was more synthesis of lipid rafts in $\mathrm{CD}^{+} \mathrm{T}$ cells of SLE patients than in normal controls, and inhibiting the synthesis of lipid rafts could alleviate the pathological manifestations of lupus in mice (35-37). Glycosphingolipids and cholesterol are important components of lipid rafts, and the levels of synthesis were significantly higher in CD4+T cells in SLE patients than in normal controls (38).

(4) Increased glutaminolysis: There is more expression of ICER, the transcriptional factor that promotes glutaminolysis and Th17 generation in $\mathrm{CD}^{+} \mathrm{T}$ cells from SLE patients than in healthy controls (39). Glutaminase 1 inhibition improved autoimmune pathology in MRL/lpr mice, and suppressed Th17 differentiation of $\mathrm{T}$ cells from patients with SLE but not in those from healthy donors (40). Those data suggested increased glutaminolysis in SLE T cells. 
(5) Highly activated mTOR: mechanistic target of rapamycin (mTOR) is a hub in the cellular metabolic signal network, regulating cellular growth and energy utilization. $\mathrm{CD}^{+} \mathrm{T}$ cells from SLE patients and lupus-prone mice showed increased mTOR activation $(32,33,41,42)$. The hyperpolarization of mitochondria and the overactivity of pentose phosphate pathway led to enhanced mTOR activity (43). In turn, highly activated mTOR can enhance glycolysis and fatty acid synthesis, thus promoting Th17 differentiation $(44,45)$. It can be seen that high mTOR expression is an important signaling mechanism leading to abnormal $\mathrm{T}$ cell metabolism and Th17/Treg imbalance in SLE patients. Many clinical trials in 2018 showed that sirolimus, an mTOR inhibitor, could alleviate the disease activity of SLE patients (46-48), expand their Foxp $3^{+}$Treg cells, and inhibit the secretion of cytokines such as IL-17 (46).

\section{green font : Metabolic pathways preferentially increased in Th17 cells \\ yellow font : Metabolic pathways preferentially increased in Tregs \\ Metabolic characteristics of SLE T cells}

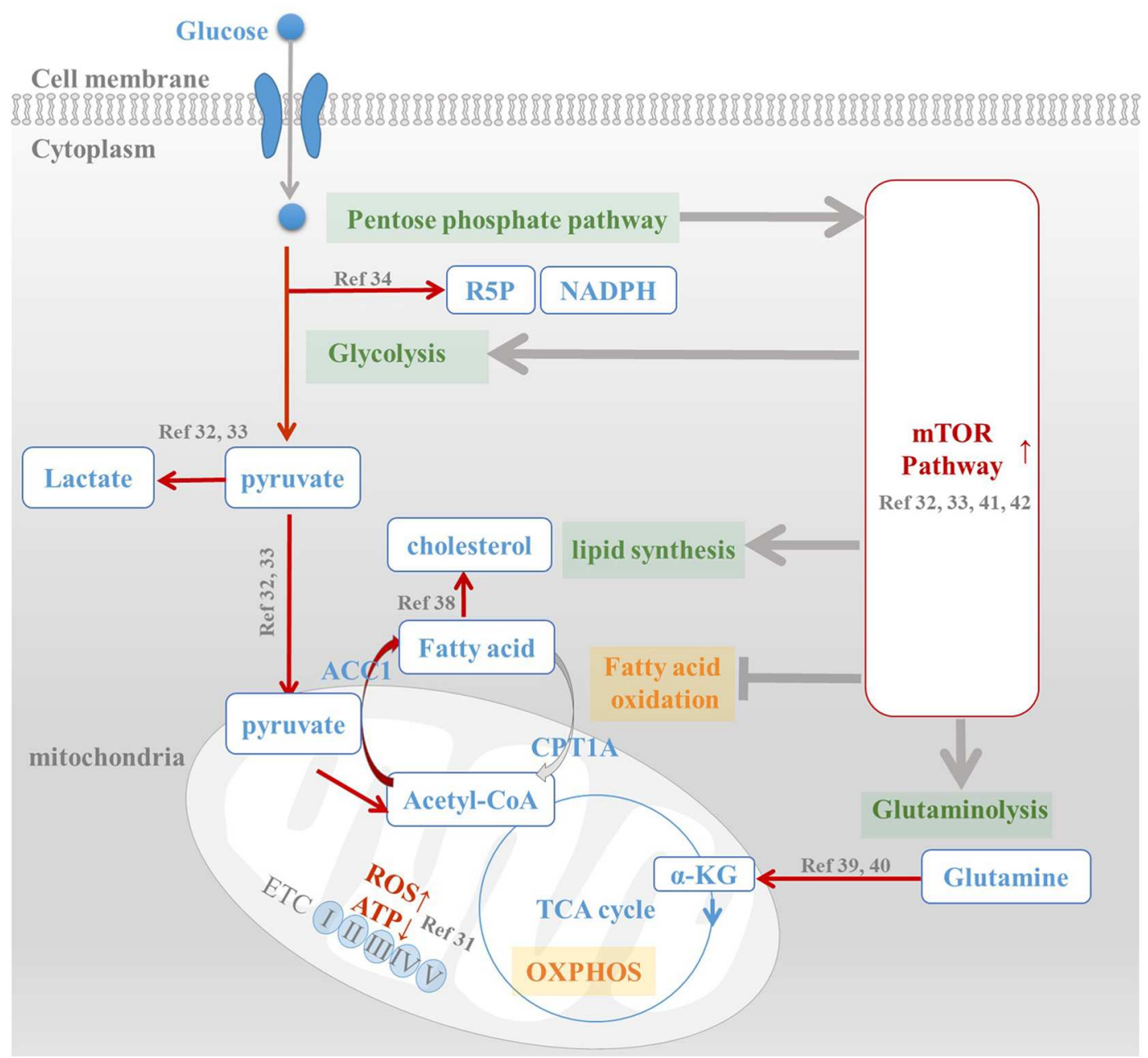

FIGURE 1 | Metabolic abnormalities is the underlying mechanism of Th17/Treg imbalance in SLE patients. Th17 and Treg cells have a distinct metabolic pattern: pentose phosphate pathway, glycolysis, fatty acid synthesis, and glutaminolysis are preferentially increased in Th17 cells, while fatty acid oxidation and oxidative phosphorylation are active in Treg cells. However, metabolic abnormalities of T cells in SLE patients, including enhanced glycolysis, active lipid synthesis, increased glutaminolysis, and high mTOR activation, are all conducive to Th17 differentiation and function. For this reason, we speculated that abnormal T cell metabolism was the mechanism underlying Th17/Treg imbalance in SLE patients. The intervention of metabolic pathways to reprogram T cell metabolic patterns in SLE patients, reduce their overactivated glycolysis and lipid synthesis levels, and promote the oxidation of fatty acids, is expected to reverse the Treg/Th17 imbalance in patients and restore their normal immune function. 


\section{DICUSSION}

The abovementioned metabolic characteristics of SLE T cells, such as enhanced glycolysis, lipid synthesis, glutaminolysis, and highly activated mTOR, all favored Th17 differentiation and function, which suggest the metabolic abnormalities of SLE T cells is the underlying mechanism of Th17/Treg imbalance in SLE patients (Figure 1). Inhibition of glycolysis $(32,33)$, lipid synthesis $(28,36-38)$, and mTOR signaling (46-48) can control inflammation and alleviate disease activity in lupus mouse and SLE patients. Manipulating cellular metabolism to correct aberrant immune responses offers promising method for SLE therapy. Further studies are still needed to explore the metabolic abnormalities occurring in T cells of SLE patients and their role in disease progression, as well as how they response to therapies, especially those have potential role in intervening Th17/Treg cell imbalance.

In addition to drugs targeting metabolic pathways, dietary habits, and nutritional factors can also modulate Th17/Treg balance by affecting $\mathrm{T}$ cell metabolism. Low cholesterol diet

\section{REFERENCES}

1. Álvarez-Rodríguez L, Martínez-Taboada V, Calvo-Alén J, Beares I, Villa I, López-Hoyos M. Altered Th17/Treg ratio in peripheral blood of systemic lupus erythematosus but not primary antiphospholipid syndrome. Front Immunol. (2019) 10:391. doi: 10.3389/fimmu.2019.00391

2. Talaat RM, Mohamed SF, Bassyouni IH, Raouf AA. Th1/Th2/Th17/Treg cytokine imbalance in systemic lupus erythematosus (SLE) patients: Correlation with disease activity. Cytokine. (2015) 72:146-53. doi: 10.1016/j.cyto.2014.12.027

3. Chen M, Chen X, Wan Q. Altered frequency of Th17 and Treg cells in new-onset systemic lupus erythematosus patients. Eur J Clin Invest. (2018) 48:e13012. doi: 10.1111/eci.13012

4. Yang J, Yang X, Zou H, Chu Y, Li M. Recovery of the immune balance between Th17 and regulatory $\mathrm{T}$ cells as a treatment for systemic lupus erythematosus. Rheumatology. (2011) 50:1366-72. doi: 10.1093/rheumatology/ker116

5. Li D, Guo B, Wu H, Tan L, Chang C, Lu Q. Interleukin-17 in systemic lupus erythematosus: a comprehensive review. Autoimmunity. (2015) 48:35361. doi: 10.3109/08916934.2015.1037441

6. Zhu Y, Huang Y, Ming B, Wu X, Chen Y, Dong L. Regulatory T-cell levels in systemic lupus erythematosus patients: a meta-analysis. Lupus. (2019) 28:445-54. doi: 10.1177/0961203319828530

7. Ohl K, Tenbrock K. Regulatory T cells in systemic lupus erythematosus. Eur J Immunol. (2015) 45:344-55. doi: 10.1002/eji.201344280

8. La Cava A. Tregs in SLE: an Update. Curr Rheumatol Rep. (2018) 20:6. doi: 10.1007/s11926-018-0714-8

9. Scalapino KJ, Daikh DI. Suppression of glomerulonephritis in NZB/NZW lupus prone mice by adoptive transfer of ex vivo expanded regulatory $\mathrm{T}$ cells. PLoS ONE. (2009) 4:e6031. doi: 10.1371/journal.pone.0006031

10. Sun L, Fu J, Zhou Y. Metabolism controls the balance of Th17/T-regulatory cells. Front Immunol. (2017) 8:1632. doi: 10.3389/fimmu.2017.01632

11. Jung J, Zeng H, Horng T. Metabolism as a guiding force for immunity. Nat Cell Biol. (2019) 21:85-93. doi: 10.1038/s41556-018-0217-x

12. Geltink RIK, Kyle RL, Pearce EL. Unraveling the complex interplay between $\mathrm{T}$ cell metabolism and function. Annu Rev Immunol. (2018) 36:46188. doi: 10.1146/annurev-immunol-042617-053019

13. Bantug GR, Galluzzi L, Kroemer G, Hess C. The spectrum of T cell metabolism in health and disease. Nat Rev Immunol. (2018) 18:1934. doi: 10.1038/nri.2017.99

14. Valerie AG, Jeffrey CR. Metabolic pathways in T cell fate and function. Trends Immunol. (2012) 33:168-73. doi: 10.1016/j.it.2012.01.010 could improve Th17/Treg balance by the activation of LXRs (49), nuclear receptors that modulate cholesterol metabolism (50). And high glucose intake was found to exacerbate autoimmunity by inducing Th17 cells via upregulation of mitochondrial ROS in $\mathrm{T}$ cells (51). The long-chain fatty acids enhanced differentiation of Th17 cells, while the short-chain fatty acids derived from a fiber-rich diet expanded Treg cells and reduce IL-17 production (52-54). Thus, a balanced diet could be helpful in the prevention and management of SLE (55).

\section{AUTHOR CONTRIBUTIONS}

JS, HJ, and YX wrote the manuscript. JS generated themes and ideas, guided, and edited the manuscript.

\section{FUNDING}

This work was supported by Natural Science Foundation of China No. 81401359.
15. Gerriets VA, Kishton RJ, Nichols AG, Macintyre AN, Inoue M, Ilkayeva O, et al. Metabolic programming and PDHK1 control CD4+ T cell subsets and inflammation. J Clin Invest. (2015) 125:194-207. doi: 10.1172/JCI 76012

16. Shi LZ, Wang R, Huang G, Vogel P, Neale G, Green DR, et al. HIF1alphadependent glycolytic pathway orchestrates a metabolic checkpoint for the differentiation of TH17 and Treg cells. J Exp Med. (2011) 208:136776. doi: 10.1084/jem.20110278

17. Kono M, Yoshida N, Maeda K, Skinner NE, Pan W, Kyttaris VC, et al. Pyruvate dehydrogenase phosphatase catalytic subunit 2 limits Th17 differentiation. PNAS. (2018) 115:9288-93. doi: 10.1073/pnas.1805717115

18. Kalim KW, Yang JQ, Li Y, Meng Y, Zheng Y, Guo F. Reciprocal regulation of glycolysis-driven Th17 pathogenicity and regulatory T cell stability by Cdc42. J Immunol. (2018) 200:2313-26. doi: 10.4049/jimmunol.1601765

19. Cluxton D, Petrasca A, Moran B, Fletcher JM. Differential regulation of human treg and Th17 cells by fatty acid synthesis and glycolysis. Front Immunol. (2019) 10:115. doi: 10.3389/fimmu.2019.00115

20. Sasaki CY, Chen G, Munk R, Eitan E, Martindale J, Longo DL, et al. p(7○S6K) in the TORC1 pathway is essential for the differentiation of Th17 Cells, but not Th1, Th2, or Treg cells in mice. Eur J Immunol. (2016) 46:21222. doi: 10.1002/eji.201445422

21. Gualdoni GA, Mayer KA, Göschl L, Boucheron N, Ellmeier W, Zlabinger GJ. The AMP analog AICAR modulates the Treg/Th17 axis through enhancement of fatty acid oxidation. FASEB J. (2016) 30:3800-9. doi: 10.1096/fj.201600522R

22. Kono M, Yoshida N, Maeda K, Tsokos GC. Transcriptional factor ICER promotes glutaminolysis and the generation of Th17 cells. Proc Natl Acad Sci USA. (2018) 115:2478-83. doi: 10.1073/pnas.1714717115

23. Xu T, Stewart KM, Wang X, Liu K, Xie M, Ryu JK, et al. Metabolic control of TH17 and induced Treg cell balance by an epigenetic mechanism. Nature. (2017) 548:228-33. doi: 10.1038/nature23475

24. Berod L, Friedrich C, Nandan A, Freitag J, Hagemann S, Harmrolfs K, et al. De novo fatty acid synthesis controls the fate between regulatory $\mathrm{T}$ and $\mathrm{T}$ helper 17 cells. Nat Med. (2014) 20:1327-33. doi: 10.1038/nm.3704

25. Young KE, Flaherty S, Woodman KM, Sharma-Walia N, Reynolds JM. Fatty acid synthase regulates the pathogenicity of Th17 cells. J Leukoc Biol. (2017) 102:1229-35. doi: 10.1189/jlb.3AB0417-159RR

26. Kidani Y, Bensinger SJ. Reviewing the impact of lipid synthetic flux on Th17 function. Curr Opin Immunol. (2017) 46:121-6. doi: 10.1016/j.coi.2017.03.012

27. Hu X, Wang Y, Hao LY, Liu X, Lesch CA, Sanchez BM, et al. Sterol metabolism controls TH17 differentiation by generating endogenous ROR $\gamma$ agonists. Nat Chem Biol. (2015) 11:141-7. doi: 10.1038/nchembio.1714 
28. Ulivieri C, Baldari CT. Statins: from cholesterol-lowering drugs to novel immunomodulators for the treatment of Th17-mediated autoimmune diseases. Pharmacol Res. (2014) 88:41-52. doi: 10.1016/j.phrs.2014.03.001

29. Morel L. Immunometabolism in systemic lupus erythematosus. Nat Rev Rheumatol. (2017) 13:280-90. doi: 10.1038/nrrheum.2017.43

30. Sharabi A, Tsokos GC. T cell metabolism: new insights in systemic lupus erythematosus pathogenesis and therapy. Nat Rev Rheumatol. (2020) 16:100112. doi: 10.1038/s41584-019-0356-x

31. Doherty E, Oaks Z, Perl A. Increased mitochondrial electron transport chain activity at complex $\mathrm{I}$ is regulated by $\mathrm{N}$-acetylcysteine in lymphocytes of patients with systemic lupus erythematosus. Antioxid Redox Signal. (2014) 21:56-65. doi: 10.1089/ars.2013.5702

32. Yin Y, Choi SC, Xu Z, Perry DJ, Seay H, Croker BP, et al. Normalization of CD4+ $\mathrm{T}$ cell metabolism reverses lupus. Sci Transl Med. (2015) 7:274ra18. doi: 10.1126/scitranslmed.aaa0835

33. Yin Y, Choi SC, Xu Z, Zeumer L, Kanda N, Croker BP, et al. Glucose oxidation is critical for CD4+ $\mathrm{T}$ cell Activation in a mouse model of systemic lupus erythematosus. J Immunol. (2016) 196:80-90. doi: 10.4049/jimmunol.1501537

34. Perl A, Hanczko R, Lai ZW, Oaks Z, Kelly R, Borsuk R, et al. Comprehensive metabolome analyses reveal $\mathrm{N}$-acetylcysteine-responsive accumulation of kynurenine in systemic lupus erythematosus: implications for activation of the mechanistic target of rapamycin. Metabolomics. (2015) 11:115774. doi: 10.1007/s11306-015-0772-0

35. Krishnan S, Nambiar MP, Warke VG, Fisher CU, Mitchell J, Delaney N, et al. Alterations in lipid raft composition and dynamics contribute to abnormal $\mathrm{T}$ cell responses in systemic lupus erythematosus. J Immunol. (2004) 172:782131. doi: 10.4049/jimmunol.172.12.7821

36. Jury EC, Isenberg DA, Mauri C, Ehrenstein MR. Atorvastatin restores Lck expression and lipid raft-associated signaling in $\mathrm{T}$ cells from patients with systemic lupus erythematosus. J Immunol. (2006) 177:741622. doi: $10.4049 /$ jimmunol.177.10.7416

37. Deng GM, Tsokos GC. Cholera toxin B accelerates disease progression in lupus-prone mice by promoting lipid raft aggregation. J Immunol. (2008) 181:4019-26. doi: 10.4049/jimmunol.181.6.4019

38. McDonald G, Deepak S, Miguel L, Hall CJ, Isenberg DA, Magee AM, et al. Normalizing glycosphingolipids restores function in CD4+ T cells from lupus patients. J Clin Invest. (2014) 124:712-24. doi: 10.1172/JCI69571

39. Yoshida N, Comte D, Mizui M, Otomo K, Rosetti F, Mayadas TN, et al. ICER is requisite for Th17 differentiation. Nat Commun. (2016) 7:12993. doi: 10.1038/ncomms12993

40. Kono M, Yoshida N, Maeda K, Suárez-Fueyo A, Kyttaris VC, Tsokos GC. Glutaminase 1 inhibition reduces glycolysis and ameliorates lupus-like disease in MRL/lpr mice and experimental autoimmune encephalomyelitis. Arthrit Rheumatol. (2019) 71:1869-78. doi: 10.1002/art.41019

41. Fernandez D, Perl A. mTOR signaling: a central pathway to pathogenesis in systemic lupus erythematosus? Discov Med. (2010) 9:173-8.

42. Kato H, Perl A. MTORC1 expands Th17 and IL-4+DN T cells and contracts Tregs in SLE. J Immunol. (2014) 192:4134-44. doi: 10.4049/jimmunol.1301859

43. Fernandez DR, Telarico T, Bonilla E, Li Q, Banerjee S, Middleton FA, et al. Activation of mammalian target of rapamycin controls the loss of TCRC in lupus $\mathrm{T}$ cells through HRES-1/Rab4-regulated lysosomal degradation. $J$ Immunol. (2009) 182:2063-73. doi: 10.4049/jimmunol.0803600
44. Zeng $\mathrm{H}$, Chi H. mTOR signaling in the differentiation and function of regulatory and effector T cells. Curr Opin Immunol. (2017) 46:10311. doi: 10.1016/j.coi.2017.04.005

45. Liu $\mathrm{Y}$, Zhang DT, Liu XG. mTOR signaling in $\mathrm{T}$ cell immunity and autoimmunity. Int Rev Immunol. (2015) 34:5066. doi: 10.3109/08830185.2014.933957

46. Lai ZW, Kelly R, Winans T, Marchena I, Shadakshari A, Yu J, et al. Sirolimus in patients with clinically active systemic lupus erythematosus resistant to, or intolerant of, conventional medications: a single-arm, open-label, phase $1 / 2$ trial. Lancet. (2018) 391:1186-96. doi: 10.1016/S0140-6736(18)30485-9

47. Yap DYH, Tang C, Chan GCW, Kwan LPY, Ma MKM, Mok MMY, et al. Longterm data on sirolimus treatment in patients with lupus nephritis. $J$ Rheumatol. (2018) 45:1663-70. doi: 10.3899/jrheum.180507

48. Herold M, Richmond NA, Montuno MA, Wesson SK, Motaparthi K. Rapamycin for refractory discoid lupus erythematosus. Dermatol Ther. (2018) 31:e12631. doi: 10.1111/dth.12631

49. Maggio R, Viscomi C, Andreozzi P, D’Ettorre G, Viscogliosi G, Barbaro B, et al. Normocaloric low cholesterol diet modulates Th17/Treg balance in patients with chronic hepatitis C virus infection. PLoS ONE. 2014 9:e112346. doi: 10.1371/journal.pone.0112346

50. Guoliang C, Qin X, Wu L, Zhang Y, Sheng X, Yu Q, et al. Liver X receptor (LXR) mediates negative regulation of mouse and human Th17 differentiation. J Clin Invest. (2011) 121:658-70. doi: 10.1172/JCI42974

51. Zhang D, Jin $\mathrm{W}, \mathrm{Wu} \mathrm{R}$, Li J, Park SA, Tu E, et al. High glucose intake exacerbates autoimmunity through reactive-oxygenspecies-mediated TGF- $\beta$ cytokine activation. Immunity. (2019) 51:671-81.e5. doi: 10.1016/j.immuni.2019.08.001

52. Haghikia A, Jörg S, Duscha A, Berg J, Manzel A, Waschbisch A, et al. Dietary fatty acids directly impact central nervous system autoimmunity via the small intestine. Immunity. (2015) 43:817-29. doi: 10.1016/j.immuni.2015.09.007

53. Mariño E, Richards JL, McLeod KH, Stanley D, Yap YA, Knight J, et al. Gut microbial metabolites limit the frequency of autoimmune $\mathrm{T}$ cells and protect against type 1 diabetes. Nat Immunol. (2017) 18:552-62. doi: 10.1038/ni.3713

54. Luu M, Pautz S, Kohl V, Singh R, Romero R, Lucas S, et al. The short-chain fatty acid pentanoate suppresses autoimmunity by modulating the metabolic-epigenetic crosstalk in lymphocytes. Nat Commun. (2019) 10:760. doi: 10.1038/s41467-019-08711-2

55. Aparicio-Soto M, Sánchez-Hidalgo M, Alarcón-de-la-Lastra C. An update on diet and nutritional factors in systemic lupus erythematosus management. Nutr Res Rev. (2017) 30:118-37. doi: 10.1017/S09544224170 00026

Conflict of Interest: The authors declare that the research was conducted in the absence of any commercial or financial relationships that could be construed as a potential conflict of interest.

Copyright (c) 2020 Shan, Jin and Xu. This is an open-access article distributed under the terms of the Creative Commons Attribution License (CC BY). The use, distribution or reproduction in other forums is permitted, provided the original author(s) and the copyright owner(s) are credited and that the original publication in this journal is cited, in accordance with accepted academic practice. No use, distribution or reproduction is permitted which does not comply with these terms. 